\title{
Historia del bocio endémico, desde Sheng-Nung hasta los programas de yodación universal de la sal en Latinoamérica
}

\author{
History of the endemic goiter, from Sheng-Nung to the \\ programs of universal yodation of salt in Latin America
}

\author{
Hernando Vargas-Uricoechea' ${ }^{1}$ LvAC, María Virginia Pinzón-Fernández ${ }^{2}{ }^{\text {CvLAC }}$, Beatriz Eugenia \\ Bastidas-Sánchez ${ }^{3}$ CvLAC
}

Fecha correspondencia:

Recibido: marzo 23 de 2018.

Revisado: mayo 8 de 2018.

Aceptado: mayo 11 de 2018.

Forma de citar:

Vargas-Uricoechea H, Pinzón-

Fernández MV, Bastidas-Sánchez

BE. Historia del bocio endémico, desde Sheng-Nung hasta los programas de yodación universal de la sal en Latinoamérica. Rev CES Med 2018; 32(2): 167-177.

Open access

(C) Derecho de autor

Licencia creative commons

Ética de publicaciones

Revisión por pares

Gestión por Open Journal System DOI: http://dx.doi.org/10.21615/

cesmedicina.32.2.10

ISSN 0120-8705

e-ISSN 2215-9177

Comparte

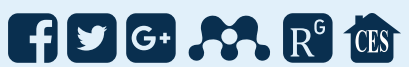

\section{Resumen}

La historia del descubrimiento del yodo y la importancia que ha tenido sobre la salud ha sido apasionante. Desde las descripciones realizadas siglos atrás, en las que se consideraba al bocio como una consecuencia de la ingesta de agua, considerar su aparición desde una perspectiva "supersticiosa", hasta establecer que su presencia era una característica de "adorno y belleza" para quienes lo padecían, mucho se ha escrito respecto a la deficiencia y al exceso de yodo en la población. Desde el momento en que Bernard Courtois descubre el yodo en 1811, las indicaciones sobre su utilidad en algunas enfermedades se extendieron casi sistemáticamente. Entre estas condiciones en salud se destacaron las infecciosas, neurológicas, reumatológicas, respiratorias, endocrinológicas, entre otras. En esta revisión abordamos los aspectos históricos más relevantes del yodo, y cómo éste se ha asociado con el desarrollo físico, social, demográfico y mental de la humanidad desde tiempos inmemoriales.

Palabras claves: Yodo; Hipotiroidismo; Hipertiroidismo; Bocio; Historia.

\begin{abstract}
The history of the discovery of iodine and the importance it has had on health has been exciting. From the descriptions made centuries ago, where goiter was considered a consequence of water intake and consider its appearance from a "superstitious" perspective, until establishing that its presence was a characteristic of "adornment and beauty" for those who suffered, much has been written about the deficiency and excess of iodine in the population. From the moment that Bernard Courtois discovered iodine in 1811, the indications about its usefulness in some diseases spread almost systematically. Among these conditions in health, infectious, neurological, rheumatological, respiratory, endocrinological, among others, stood out. In this review, we address the most relevant sequential historical aspects of iodine, and how it has been associated with the physical, social, demographic and mental development of humanity from time immemorial.
\end{abstract}

Keywords: lodine; Hypothyroidism; Hyperthyroidism; Goiter; History. 
Mayo - Agosto 2018 - Pág 168

\section{Sobre los autores:}

1. Especialista en medicina interna y endocrinología, MSc en epidemiología, Ph. Dc. en ciencias biomédicas, profesor asociado Universidad del Cauca, Departamento de medicina interna.

2. Ph.Dc, Antropología. MSc en Salud Pública, Bacterióloga, especialista en Educación. Profesora Titular, Universidad del Cauca, Departamento de medicina interna.

3. Médica especialista en Salud Familiar y Epidemiología, Profesora titular departamento de Medicina Social y Salud Familiar, Universidad del Cauca.

En el Imperio Bizantino, el arte de la pintura sacra representa frecuentemente la presencia de bocio. En la Edad Media, Abu al-Qasim (siglo X) diferencia el bocio congénito del adquirido y se le atribuye la primera realización de biopsia con aguja y la primera tiroidectomía.
El presente es un artículo de contexto histórico, cuyo objetivo principal es realizar una síntesis de los aspectos más relevantes de la historia del bocio endémico y de la forma como esta condición ha acompañado a la humanidad desde sus inicios. Para ello se consultaron fuentes, artículos históricos originales, y de discusión sobre el tema, al igual que bases de datos de carácter histórico, periódicos y archivos relacionados.

\section{La historia}

Las primeras referencias de bocio en la Edad Antigua se atribuyen al emperador chino Shen-Nung (2838-2898 a.C.) quién, en su "Tratado de las hierbas y de las raíces" menciona el alga marina Sargassum como remedio eficaz para esta condición (1- $\underline{3}$ ). En el libro de Shan Hkai Tsing (770-220 a.C.) "Tratado de las aguas y de las hierbas", se atribuye esta enfermedad a la mala calidad del agua. Referencias posteriores describen como factores etiológicos las "emociones profundas" y determinadas condiciones de vida en las zonas montañosas; en la antigua China se llegaron a utilizar fragmentos macerados de tiroides de animales para el tratamiento del bocio $(\underline{4}, \underline{5})$.

Los hindúes conocían esta enfermedad y hacen referencia a ella en el Atharva-Veda (2000 a.C.) en donde aparecen "fórmulas mágicas" contra el bocio; los antiguos médicos hindúes dieron el nombre de galaganda a los tumores del cuello. En el Papiro de Ebers (1500 a.C.) se describen los "tumores del cuello" y eran tratados quirúrgicamente (ㅁ-요). Los escritos hipocráticos (siglo IV a.C.) se refieren al bocio como joiron, botium o gongrona. En el conocido tratado hipocrático "De aires, aguas y lugares" se considera al agua como la causa del bocio. Los romanos describen la prevalencia de bocio endémico en Los Alpes y se les atribuye la observación de que el engrosamiento del cuello era una característica de los galos $(\underline{9}, \underline{10})$.

En el siglo I de la era cristiana Plinio describe el bocio así: "La inflamación del cuello se da sólo en los hombres y los cerdos y la motiva el agua que beben". En Bohemia y Alemania se cree que el bocio es provocado por un trabajo agotador o por ataques frecuentes de tos, o que aparece en una mujer después de un parto difícil o por los rayos de la luna. En el Imperio Bizantino, el arte de la pintura sacra representa frecuentemente la presencia de bocio (figuras 1 y 2). En la Edad Media, Abu al-Qasim (siglo X) diferencia el bocio congénito del adquirido y se le atribuye la primera realización de biopsia con aguja y la primera tiroidectomía (11).

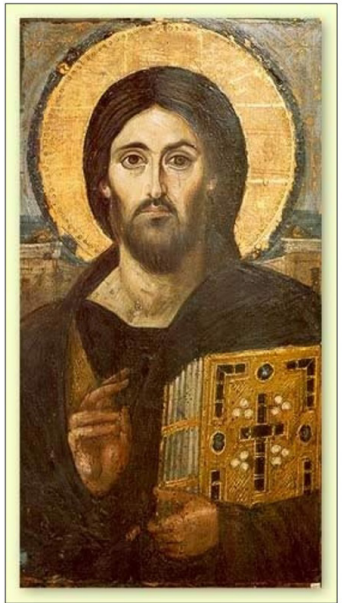

Figura 1. El Pantocrátor de Santa Catalina, Siglo VI. Monasterio de Santa Catalina, Monte Sinaí (Egipto). Nótese el abultamiento en la región anterior del cuello, de predominio derecho, sugiriendo la presencia de bocio (imagen tomada de ref. 12). 
Mayo - Agosto 2018 - Pág 169

En 1718, Lorenz Heister publica su libro "Chirurgie" donde describe el manejo quirúrgico y no quirúrgico del bocio.

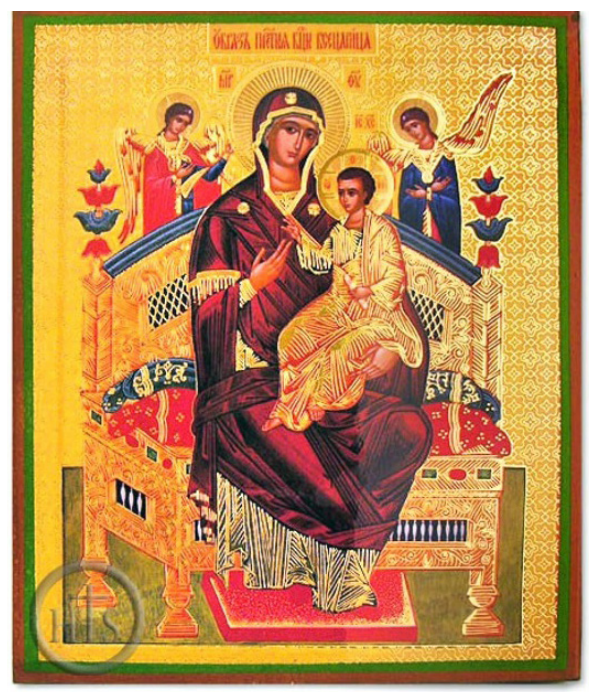

Figura 2. El icono original de la Madre de Dios, llamado "Vsetsaritsa (Pantanassa)" Iglesia del monasterio de Vatopedsk (Santo Monte Athos, Grecia). Se aprecia abultamiento en la región anterior del cuello tanto en la Virgen, como en El Niño y en los Ángeles, sugiriendo la presencia de bocio (imagen tomada de ref. 13).

En el siglo XIII, Marco Polo observa bocio en Yakarta. En el Renacimiento, Paracelso lo atribuye a la falta de minerales en el agua, y se describen endemias en los cantones de Valais, los Grisones, Utervaz, Zizers e Igis, en la cuenca del Lena, Rusia, Polonia, Ginebra e Italia. En 1610, Jessenius describe que la gente considera el bocio como una forma de adorno (14). En el siglo XVIII, Sausure de Ginebra hace una notable descripción del cretinismo endémico, aunque algunos médicos atribuyen el cuadro a un raquitismo avanzado.

El término "Struma" es descrito por primera vez por Albrecht von Haller. En 1796, Foderé observa casos de cretinismo en Saboya y el valle de Aosta (Francia), pero rechaza la etiología raquítica del proceso. En la primera mitad del siglo XVIII, el bocio endémico se conocía en Inglaterra como "cuello de Derbyshire" y Vesalio describe el tiroides como "un compuesto por dos glándulas situadas a ambos lados de la raíz de la laringe". Eustaquio descubre el istmo tiroideo y Wharton describe la anatomía, el tamaño y peso de la glándula.

En 1718, Lorenz Heister publica su libro "Chirurgie" donde describe el manejo quirúrgico y no quirúrgico del bocio (15). Schreger fue el primero en describir la especial irrigación sanguínea del tiroides y Luschka considera que la glándula tiroidea es una almohadilla que protege la laringe, tráquea, vasos sanguíneos y nervios del cuello contra la presión muscular. A finales del siglo XVIII, Albrecht von Haller clasifica el tiroides, el timo y el bazo como glándulas de secreción interna y sin conducto excretor; sin embargo, la función de esta glándula no llegaría a comprenderse hasta el último decenio del siglo XIX (16).

El conocimiento de la hipofunción tiroidea o mixedema es todavía más reciente: Gull en 1873 y Ord en 1878, describen el mixedema como "un estado cretinoide que se manifiesta en la mujer durante la edad adulta". Entre 1882-1883, los cirujanos Reverdin y Kocher reconocen la "caquexia estrumipriva" o hipotiroidismo consecuente a la tiroidectomía, lo que constituye el aporte definitivo para establecer la importancia del tiroides en la homeostasis interna. 
Mayo - Agosto 2018 - Pág 170

La teoría que el bocio endémico se debe a la deficiencia de yodo la formula por primera vez Jean Louis Prévost, junto al italiano Maffoni en 1846. El yodo fue descubierto en 1811 por Bernard Courtois.
En ese siglo los informes sobre el bocio endémico se multiplican: en 1845, el rey Carlos Alberto de Cerdeña nombra una comisión encargada de estudiar el alcance del bocio endémico y de recomendar las medidas sanitarias para combatirlo. En 1864, el gobierno francés establece una comisión análoga, que en 1874 determina que en Francia había 370403 sujetos mayores de 20 años con bocio, y alrededor de 120000 cretinos e idiotas (Francia tenía 36 millones de habitantes).

La teoría que el bocio endémico se debe a la deficiencia de yodo la formula por primera vez Jean Louis Prévost, junto al italiano Maffoni en 1846. Entre 1850-1851, el químico francés Chatin publica una serie de trabajos en los que encuentra que la incidencia de bocio es inversamente proporcional a la riqueza de yodo en las aguas del medio. En 1895, Baumann demuestra que la tiroides alberga una gran cantidad de yodo y aísla una sustancia a la que denominó "tiroidina" $(\underline{17}, 18)$.

Ya en el siglo XX, Oswald aísla la tiroglobulina y Kendall la tiroxina cristalina (sintetizada después por Harrington). Estos descubrimientos demuestran la importancia del yodo en la función tiroidea y renuevan el interés por la terapéutica y profilaxis yódicas $(\underline{17}, \underline{18})$.

\section{Descubrimiento del yodo y Bernard Courtois}

El yodo fue descubierto en 1811 por Bernard Courtois. Para entonces, Francia estaba en guerra con sus vecinos y necesitaba disponer de cantidades sustanciales de pólvora para su uso militar. La gran demanda del nitrato potásico como componente mayoritario de la pólvora conduce a la explotación de plantaciones de salitre, lo que requería disponer de una gran cantidad de carbonato sódico. Courtois ejerce durante tres años como aprendiz de farmacia en Auxerre y en 1799 es llamado a filas para el ejército de Napoleón, con el propósito de servir como farmacéutico en hospitales militares. Como a lo largo de las costas y a escasa profundidad se encontraban plantas marinas, Courtois obtiene las aguas madres y las sales sódicas o potásicas por medio de calor, y una vez se produce la evaporación, precipita el cloruro sódico y el cloruro y sulfato potásicos. De esta forma, en una ocasión, Courtois observa la formación de nubes de vapor violetas, con un olor irritante parecido al del cloro, que se condensan sobre objetos fríos, originando cristales oscuros de lustre acerado y metálico, estableciendo entonces la presencia de un nuevo elemento químico $(\underline{19}, \underline{20})$.

Posteriormente, la naturaleza elemental y la síntesis de varios productos de yodo y sus características son demostradas por Davy y Gay Lussac en 1813. Gay Lussac le da todo el crédito a Courtois por el descubrimiento del yodo, aunque Davy reclama en su momento la caracterización del yodo (intentando opacar el trabajo de Gay Lussac). En realidad, Courtois no se beneficia de su descubrimiento, y aunque recibe un premio de 6000 francos, termina ganándose la vida preparando y vendiendo compuestos de yodo y viviendo más en la pobreza que en la opulencia (21).

En 1819, Coindet establece que el yodo puede ser el ingrediente activo en las algas marinas. Probablemente, la forma de obtención del yodo parece haberse establecido décadas antes del descubrimiento de Courtois (a través de la reducción de algas marinas, mediada por calor); por ejemplo, en el libro The third edition of the London Pharmacopeia, de 1751, se describe lo siguiente: "Caliente la esponja en un recipiente cubierto hasta que se vuelva negra y fácilmente friable; posteriormente, reduzca a polvo en un mortero de vidrio o mármol. El calor utilizado en ese momento debe ser mucho mayor que en el proceso anterior; pero, se debe tener cuidado de no quemar la esponja hasta que su sal volátil sea expulsada, pues así lo reduciría a un mero carbón". 
Mayo - Agosto 2018 - Pág 171

En 1831, Boussingault propone la yodación del cloruro de sodio (tabletas de sal) como medida preventiva del bocio. Esta medida se implementa inicialmente en Europa y posteriormente en Estados Unidos en la década de 1920.
Mas adelante, en el libro "Lewis Materia Medica, London, 1768" se describe: "Queme la esponja en un recipiente cerrado de barro hasta que se vuelva negra y friable. De esta forma se ha dado para las escrófulas y defectos cutáneos, en la que a veces ha sido útil, en virtud, probablemente, de su materia salina, cuya proporción, después de la gran reducción que la esponja ha sufrido en la quema"

Estas dos descripciones fueron las que tuvo en cuenta Courtois como protocolo en la síntesis del nitrato de potasio (como componente mayoritario de la pólvora y que llevó indirectamente al descubrimiento del yodo). En 1819, Coindet evalúa el efecto de la solución de yodo en dosis de $250 \mathrm{mg} /$ día en 150 pacientes con bocio (reduciendo el tamaño del bocio en un lapso de una semana). Coindet publica sus resultados en 1820 , y a pesar de que no se hacen cuestionamientos con la cantidad excesiva de yodo utilizada, la historia reconoce que es el pionero en usar el yodo en la práctica clínica (22).

Adicionalmente, Coindet describe los efectos adversos secundarios del tratamiento, e incluso, describe hallazgos de hipertiroidismo inducido por yodo. En 1821, Francois Magendie sitúa el yodo en la farmacopea, convirtiéndose en un remedio de moda para las "enfermedades escrófulas" y como tal, Christison, en 1848 afirma: "La esponja contiene un rastro combinado de yodo, y antes del descubrimiento de este elemento y sus compuestos, en el estado quemado, era un remedio para la escrófula y el bocio. Su uso interno, sin embargo, ahora es obsoleto".

A causa de que el yodo no es muy soluble en agua (330 mg/L), las llamadas "tinturas" con alcohol se indicaban para uso externo (como antisépticas) y para uso interno (como tratamiento para ciertas condiciones médicas y quirúrgicas). En 1829 , Jean Lugol evalúa la utilidad del yodo en enfermedades infecciosas y establece que la solución de "lugol" debería contener $5 \%$ de yodo y $10 \%$ de yoduro de potasio. Lugol utiliza esta mezcla para el tratamiento de lo que en su momento se denominaba "les maladies scrofuleuses" (las cuales incluían, entre otras, a la sífilis, corea, gota, fístulas lacrimales, gangrena, carbunco, panadizo herpético, lupus, asma, ántrax y bronquitis) y demuestra que el suministro en la dosis adecuada originaba una mejor respuesta clínica con menor tasa de complicaciones que el uso aislado de yodo o de yoduro $(\underline{23}, \underline{24})$.

Con el tiempo, se establece el concepto que los "yoduros" no tienen propiedades antisépticas ni antibacterianas, y que únicamente el yodo (el estado oxidado) tiene propiedades antisépticas. A partir de esta experiencia, los médicos recomiendan una dosis de 0,1 a 0,3 mL de solución de lugol (que contiene entre 12,5-37,5 mg de yodo elemental) para el suplemento de yodo/yoduro.

En 1831, Boussingault propone la yodación del cloruro de sodio (tabletas de sal) como medida preventiva del bocio. Esta medida se implementa inicialmente en Europa y posteriormente en Estados Unidos en la década de 1920; sin embargo, dio una falsa sensación de "suficiencia" de yodo, puesto que, para poder suplementar $12,5 \mathrm{mg}$ de yodo elemental a partir de las tabletas de sal, los individuos debían consumir $165 \mathrm{gm}$ de sal (y el triple de esta dosis en caso de requerir suplementar 37,5 mg de yodo elemental). Entre 1891 y 1892, se reporta por primera vez el uso eficaz de extractos de tiroides por vía oral y parenteral en individuos con hipotiroidismo. En 1895, Bauman detecta altas concentraciones de yodo en el tiroides y propone que el componente activo en los extractos tiroideos es el yodo (25). 
Mayo - Agosto 2018 - Pág 172

Posterior a estos trabajos, la suficiencia de yodo se asocia con la "ausencia de bocio" y como resultado, la medida de salud pública que se toma para el control del bocio poblacional es el establecimiento de la yodación por medio del suministro de tabletas de sal.

\section{Uso del yodo a lo largo del siglo XX}

En el siglo XX, el yodo estaba bien establecido en las prácticas médicas y quirúrgicas, como se describe en "The Encyclopedia Britannica 11 th Edition, 1910-1911": "En la forma de ciertas sales el yodo es muy utilizado para ser administrado en el tratamiento de muchas condiciones usualmente clasificadas como quirúrgicas, tales como las manifestaciones óseas de la sífilis terciaria. La sal más comúnmente utilizada es el yoduro de potasio; los yoduros de sodio y amonio son casi tan frecuentemente utilizados, y los de calcio y estroncio son de uso ocasional". Para la fecha, también se comercializan vendas con yodo, las cuales se colocan alrededor del cuello para el tratamiento del bocio (26).

En los años 10's y 20's se destacan los estudios de Marine et al., quienes reportan un efecto positivo de la suplementación de yodo en dosis de $9 \mathrm{mg} /$ día para la prevención del bocio simple en mujeres adolescentes; dicha dosis está basada en investigaciones previas en animales de granja y de laboratorio. No obstante, en los estudios de Marine, el único parámetro evaluado es la presencia de bocio. Posterior a estos trabajos, la suficiencia de yodo se asocia con la "ausencia de bocio" y como resultado, la medida de salud pública que se toma para el control del bocio poblacional es el establecimiento de la yodación por medio del suministro de tabletas de sal (27).

Esta decisión es implementada exitosamente en Estados Unidos entre 1917 y 1924 (lo que origina una disminución significativa en la incidencia de bocio simple). En 1927, en España, Gregorio Marañón en su obra El bocio y el cretinismo escribe: "...establecer la morbilidad de la endemia bociosa resulta difícil porque son sujetos que solo acuden al médico cuando padecen cuadros muy avanzados y llamativos, de no ser así no suelen consultar, porque no se consideran enfermos o por habitar en zonas muy alejadas de los centros sanitarios" Marañón concluye que la "entidad morbosa" afecta entre 10000 a 15000 individuos (0,006 \% de la población española).

Con la posterior disponibilidad de las hormonas tiroideas en los años 30's, el manejo con yodo para el bocio endémico es ignorado por los clínicos de la época, y en el libro Diagnosis and treatment of disease of the thyroid (Rowland, 1932) se menciona explícitamente que la causa más común de hipotiroidismo y bocio simple es la deficiencia de yodo. Este concepto determina la recomendación de suministrar extractos tiroideos en individuos con deficiencia de yodo y bocio (en vez del uso de preparaciones que contenían yodo/yoduro) $(\underline{27}, \underline{28})$.

Estas consideraciones conllevan en los años 40's y a principios de los 50's a la falsa creencia que la ausencia de bocio significa "suficiencia" de yodo, y origina que los textos sobre enfermedad tiroidea no describan el fenómeno de la deficiencia de yodo y del bocio endémico; incluso, en Estados Unidos, después de la Segunda Guerra Mundial se retiran del mercado muchos suplementos que contienen yodo. Este temor a los efectos del yodo sobre la salud se denominó "yodofobia".

Un determinante en la aparición de la yodofobia fueron los estudios de Wolff y Chaikoff en 1948, quienes encuentran que, al administrar yoduro por vía intraperitoneal en roedores, al llegar a un nivel sérico de yoduro inorgánico de 0,2 mg/L, la captación de yoduro por parte del tiroides es indetectable, indicando que la suficiencia de yodo en la glándula tiroidea se alcanza cuando los niveles de yoduro inorgánico están en dicho nivel. Por tanto, concluyen que los niveles séricos de yoduro inorgánico en dicha concentración bloquean la síntesis de hormona tiroidea, causando hipotiroidismo y bocio (aunque realmente no titularon los niveles de hormonas tiroideas y tampoco 
Mayo - Agosto 2018 - Pág 173

Entre las décadas de los 50's y 60's, casi todos los países del área se preocupan por crear leyes que regulen la yodación de la sal para consumo humano. se observó hipotiroidismo o bocio en los roedores). Al final, los resultados de dicho estudio se extrapolan a los humanos; a este fenómeno ficticio se le denomina "efecto de Wolff y Chaikoff".

En 1970 Wartofsky evalúa el efecto de la solución de Lugol en individuos con tirotoxicosis y concluye que la disminución abrupta en la secreción de T4 inducida por yodo no es secundaria a la inhibición aguda y sostenida de la síntesis de T4, sino que es más probable que se deba a la disminución abrupta en la tasa fraccional de la liberación de T4; este impacto yodofóbico en la comunidad científica y en la sociedad se denomina "efecto dominó yodofóbico de Wolff y Chaikoff" $(\underline{29}, \underline{30})$.

\section{El bocio en la República de la Nueva Granada y en Colombia}

El vocablo bocio es derivado del bajo latín al francés medioeval bosse, y que fue cambiado al de coto, palabra que tiene su origen en el vocablo quechua Kcoto, (basado en la raíz lingüística del quechua y del aymará, cuyas voces ccoto o kcoto podrían haber estado asociadas más al significado de "montón", "buche" o papada). En Colombia, las primeras menciones sobre el bocio son las de José Celestino Mutis en su Diario de observaciones (1761-1762); sin embargo, los desórdenes por deficiencia de yodo se documentaron a partir de 1794, en un artículo anónimo titulado: Reflexiones sobre la enfermedad que vulgarmente se llama coto y publicado en el Papel Periódico de Santa Fe de Bogotá (1794). Esta publicación contiene una nota del redactor en la que se hace una excepcional descripción clínica del bocio. Por otro lado, el bocio fue una preocupación para Francisco José de Caldas, quien promueve la creación de un premio especial para resolver un problema de tanta importancia para la salud $(\underline{31}, \underline{32})$.

Ya en el siglo XX y a finales de la década de los 40 se realiza el primer gran estudio sobre la prevalencia de bocio endémico en Colombia (encontrando que era del $53 \%$, aunque en algunos sitios fue mayor del 80 \%). En 1947 se establece la yodación de la sal para consumo humano y en 1960 se determina que la prevalencia de bocio endémico es de $39,5 \%$ en hombres y de $43,7 \%$ en mujeres. La eficacia de la intervención poblacional a través de la yodación de la sal se comprueba posteriormente (hacia principio de los años 80 's) cuando la prevalencia fue menor de $2 \%$.

Al comparar el estado poblacional del yodo con otros países, en la década de los 30's prácticamente ningún país de Latinoamérica se encontraba libre de desórdenes por deficiencia de yodo; las encuestas demostraban que casi todos los países tenían regiones donde la prevalencia de bocio era mayor del 50 \%, y Argentina, Bolivia, Brasil, Ecuador, Paraguay, Perú, México y Guatemala, tenían deficiencia de yodo en la mayor parte de su territorio.

Entre las décadas de los 50's y 60's, casi todos los países del área se preocupan por crear leyes que regulen la yodación de la sal para consumo humano; no obstante, los rangos de yodación que se establecieron fueron muy amplios y arbitrarios (33).

En algunos países la profilaxis con sal yodada fue transitoriamente exitosa, pero en la mayoría no fue así; incluso, el éxito inicial de algunos programas es opacado con el tiempo, debido a que las leyes no se cumplieron a cabalidad, o bien el monitoreo posterior no fue adecuado, o la importancia de los desórdenes por deficiencia de yodo y su intervención no fue comunicada a los actores correspondientes. Como consecuencia, en la década de los 90's sólo algunos países se aproximaron a la definición de "suficiencia de yodo" y, por ende, la prevalencia de bocio apenas se había modificado (cuadro 1). 
Mayo - Agosto 2018 - Pág 174

Cuadro 1. Prevalencia de bocio endémico en Latinoamérica antes y después de la introducción de la legislación de la sal yodada (1940-1970) y mediana de yoduria entre los años 2002 y 2016

\begin{tabular}{|c|c|c|c|c|c|}
\hline País & $\begin{array}{c}\text { Año de introducción } \\
\text { de legislación de la } \\
\text { sal yodada }\end{array}$ & $\begin{array}{c}\text { Yodación } \\
\text { (ppm) }\end{array}$ & $\begin{array}{c}\text { Prevalencia (\%) de } \\
\text { bocio endémico } \\
(1950-1960)\end{array}$ & $\begin{array}{c}\text { Prevalencia (\%) de } \\
\text { bocio endémico } \\
\text { (1980-1990) }\end{array}$ & $\begin{array}{c}\text { Mediana de la } \\
\text { concentración de } \\
\text { yoduria }(\mu g / L)^{*}\end{array}$ \\
\hline Argentina & 1967 & $20-40$ & $34-83$ & $5-42$ & 123 \\
\hline Bolivia & 1968 & $40-80$ & 68 & 61 & 191 \\
\hline Brasil & 1953 & $40-60$ & 27 & $29-35$ & 277 \\
\hline Chile & 1959 & 100 & 25 & 10 & 252 \\
\hline Colombia & 1955 & $50-100$ & 53 & 14 & 415 \\
\hline Costa Rica & 1941 & $33-50$ & 19 & 4 & 314 \\
\hline Ecuador & 1968 & $50-100$ & 34 & 37 & 162 \\
\hline El Salvador & 1961 & $30-100$ & 30 & 25 & 206 \\
\hline Guatemala & 1954 & $30-100$ & 38 & 20 & 144 \\
\hline Honduras & 1960 & $50-100$ & 22 & 9 & 356 \\
\hline México & 1963 & $20-40$ & $5-46$ & $5-50$ & 297 \\
\hline Nicaragua & 1969 & $30-100$ & 27 & 20 & 196 \\
\hline Panamá & 1955 & $66-100$ & 17 & 13 & 291 \\
\hline Paraguay & 1958 & $40-60$ & 50 & 49 & 296 \\
\hline Perú & 1969 & $30-40$ & 28 & 36 & 262 \\
\hline Uruguay & 1961 & $30-40$ & $7-38$ & 9 & 248 \\
\hline Venezuela & 1968 & $35-60$ & 33 & 33 & 180 \\
\hline
\end{tabular}

*Datos procedentes de encuestas realizadas en escolares (OMS 2002-2016). Ref. 34.

Como resultado de los esfuerzos realizados por el Estado, las instituciones y organizaciones internacionales, el 29 de abril de 1998 se llevó a cabo el acto de declaratoria de Colombia como país "libre de desórdenes por deficiencia de yodo".
En 1990 se lleva a cabo la Cumbre Mundial por la Infancia de las Naciones Unidas, y se establece la eliminación de los desórdenes por deficiencia de yodo como un problema de salud pública para el año 2000. En 1993, la Organización Mundial de la Salud y el Fondo Internacional de Emergencia de las Naciones Unidas para la Infancia (UNICEF) recomiendan la yodación universal de la sal como la principal estrategia para lograr la eliminación sostenida de los desórdenes por deficiencia de yodo. En 1996, la Asamblea Mundial de la Salud adopta la resolución WHA58.24, que compromete a los países a informar cada tres años sobre la situación mundial de los desórdenes por deficiencia de yodo. Como resultado de los esfuerzos realizados por el Estado, las instituciones y organizaciones internacionales, el 29 de abril de 1998 se llevó a cabo el acto de declaratoria de Colombia como país "libre de desórdenes por deficiencia de yodo".

Finalmente, un comité de expertos de la OMS, la UNICEF y el Consejo internacional para el control de los desórdenes por deficiencia de yodo en 2007, definen los indicadores epidemiológicos del estado nutricional de yodo en base a la ingesta estimada y la concentración de yodo en orina, estableciendo los valores de referencia (cuadro 2) (35).

Cuadro 2. Recomendaciones de ingesta diaria de yodo de acuerdo al grupo de riesgo (ref. 35)

\begin{tabular}{ll}
\hline Edad & Ingesta diaria recomendada \\
\hline Niños en etapa preescolar (0-59 meses) & $90 \mu \mathrm{g}$ \\
\hline Niños en etapa escolar (6-12 años) & $150 \mu \mathrm{g}$ \\
\hline Adolescentes (>12 años) & $150 \mu \mathrm{g}$ \\
\hline Adultos & $100-300 \mu \mathrm{g}$ \\
\hline Mujeres en embarazo y en lactancia & $250 \mu \mathrm{g}$ \\
\hline
\end{tabular}




\section{Consideraciones finales}

El bocio y los desórdenes por deficiencia de yodo han acompañado a la humanidad desde sus comienzos. Aspectos como los compromisos gubernamentales, el apoyo de la industria de la sal, el uso de sal yodada por parte de la industria alimentaria, el monitoreo regular del estado del yodo poblacional, el conocer las consecuencias de la deficiencia de yodo (y el exceso en el consumo de sal) han permitido que la eliminación de los desórdenes por deficiencia de yodo sea considerada como un logro indiscutible de las políticas de salud pública.

\section{Bibliografía}

1. Hetzel BS. The story of iodine deficiency, an international challenge in nutrition. New Delhi: Oxford University Press, 1989. pp. 36-51.

2. Langer P. History of Goitre. World Health Organization. Monograph Series No. 44, 1960.p:9-26.

El bocio y los desórdenes por deficiencia de yodo han acompañado a la humanidad desde sus comienzos. Aspectos tales como el apoyo estatal y de la industria alimenticia, acompañados de programas de salud pública, han permitido que la eliminación de los desórdenes por deficiencia de yodo sea considerada como un logro indiscutible en muchos países.
3. Iodine. In: Encyclopedia Britannica, 11th Edition, 1910-1911, Vol. XIV. 725-726.

4. Abraham GE. The historical background of the iodine project. The Original Internist. 2005;12(2):57-66.

5. Miles M. Goitre, cretinism and iodine in South Asia: historical perspectives on a continuing scourge. Med Hist. 1998;42:47-67.

6. Iason AH. The Thyroid Gland in Medical History. New York, NY: Froben; 1946.

7. Niazi AK, Kalra S, Irfan A, Islam A. Thyroidology over the ages. Indian J Endocr Metab. 2011:15(S2):121-126.

8. Cranefield PF. The discovery of cretinism. Bull Hist Med. 1962;36:489-511.

9. Lindholm J, Laurberg P. Hypothyroidism and Thyroid Substitution: Historical Aspects. J Thyroid Res. 2011; 2011:809341. doi: 10.4061/2011/809341.

10. Pharoah PD, Delange F, Fierro Benitez R. In Stanbury JB \& Hetzel BS (eds.). Endemic goitre and endemic Cretinism. New York: Wiley, 1980, pp. 395-451.

11. Medvei VC, Medvei VC. (1993). The history of clinical endocrinology: A comprehensive account of endocrinology from earliest times to the present day. Carnforth, Lancs., UK: Parthenon Pub. Group.

12. Anónimo, Dominio público. http://campus.belmont.edu/honors/Sinailcons/Sinailcons.html Acceso abril 18 de 2018.

13. Dominio público. https://i.pinimg.com/564x/38/cd/4b/38cd4b2d04b31aca3be5a59aa201b64a.jpg Acceso Noviembre 12, 2017.

14. Józsa LG. Goiter depicted in Byzantine artworks. Hormones. 2010;9(4):343-346.

15. Merke F. The history of endemic goitre and cretinism in the thirteenth to fifteenth centuries. Proc R Soc Med. 1960;53:995-1002. 
16. Merkel F. (1984) History of Endemic Cretinism in: Merke F., Editor History and Iconography of Endemic Goiter and Cretinism, Hans Huber publisher; Berne, Switzerland 1984; pp:195-233.

17. Greenwald I. The significance of the History of goiter for the etiology of the Disease. Am J Clin Nutr. 1960;8:801-807.

18. Cap PA. Notes Historiques sur Bernard Courtois et Sur la Découverte de l'lode. J. Pharmacie et Chemie. 1851;20:131-138.

19. Raubenheimer 0. The Centenary of The Discovery of lodine. J Pharm Sci. 1913;2(12):1547-1551.

20. Guyotjeannin C. In memory of Bernard Courtois (1777-1838). Rev Hist Pharm (Paris). 1995;42(305):117-123.

21. Crosland M. Gay-Lussac (1778-1850): A view of chemistry, industry and society in post-revolutionary France. Endeavour. 1978;2(2):52-56.

22. Coindet JF. Découverte d'un nouveau remède contre le goıtre. Ann. Chim. Phys. 1820; 15(Ser. 2):49-59.

23. Carpenter KJ. David Marine and the problem of goiter. J Nutr. 2005;135(4):675-680.

24. Abraham GE. The History of lodine in medicine. Part 1: From discovery to essentiality. The Original Internist. 2006;13(1):29-36.

25. Lugol, J. G. A., Mémoire sur l'emploi de l'iode dans les maladies scrophuleuses, suivi d'un précis sur lárt de formuler les preparations ioudrées, 1,219. París: J.B. Ballière, 1830.

26. Marine D, Williams WW. The relation of iodine to the structure of the thyroid gland. Arch Intern Med. 1908;1:349-384.

27. Rosenfeld, L. Discovery and early uses of iodine. J Chem Educ. 2000;77:984-987.

28. Marine D and Kimball OP. Prevention of simple goiter in man. Arch Intern Med.1920;25:661.

29. Marañón, Gregorio: El bocio y el cretinismo. Madrid, 1.927, p. 22.

30. Wolff J, Chaikoff IL. Plasma inorganic iodide as a homeostatic regulator of thyroid function. J Biol Chem. 1948;174(2):555-564.

31. Wartofsky L and Ingbar SH. Estimation of the rate of reléase of non-thyroxine iodine from the thyroid glands of normal subjects and patients with thyrotoxicosis. J Clin Endocrinol Metab. 1971;33(3):488-500.

32. Anónimo: Reflexiones sobre la enfermedad que vulgarmente se llama Coto. Papel Periódico de Santa Fé de Bogotá. (137):669-676, Viernes 11 de Abril de 1794. 
33. Greenwald I.The history of goiter in Venezuela, Colombia and Ecuador. Tex Rep Biol Med.1971;29(4):451-465.

34. lodine Global Network. Global Scorecard of lodine Nutrition in 2017 in the general population and in pregnant women (PW). IGN: Zurich, Switzerland. 2017.

35. Vargas-Uricoechea H, Sierra-Torres CH, Holguín-Betancourt CM, Cristancho-Torres L. Trastornos asociados a la deficiencia de yodo. Vigilancia permanente es deficitaria en zonas vulnerables. MEDICINA (Bogotá). 2012;34 (2):119-144. 